\title{
Administration der
}

\section{Härtefallregelungen in der}

\section{Gesetzlichen Krankenversicherung - Eine empirische Analyse}

PROF. DR. BERNHARD

LANGER,

ELISABETH LANGER, B.SC., JULIANE MÜLLER, B.SC., FRANZISKA PAUL, B.SC., CHRISTIN

STROHSCHNEIDER, B.SC.

Die Autoren sind Wissenschaftler an der Hochschule Neubrandenburg im Fachbereich Gesundheit, Pflege, Management

Da die Erhebung von Zuzahlungen für Geringverdiener bzw. Versicherte mit einem schlechten Gesundheitszustand und demzufolge hohen Leistungsbedarf sehr schnell zu einer übermäßigen Belastung führen kann, wurden beginnend mit dem Jahr 1989 Zuzahlungsbefreiungen - so genannte Härtefallregelungen - gesetzlich verankert. Die wenigen bislang durchgeführten Forschungsvorhaben ließen die administrative Umsetzung der Härtefallregelungen durch die Gesetzlichen Krankenkassen sowie deren mögliches Optimierungspotential weitgehend unberücksichtigt. Diese Lücke versucht der vorliegende Beitrag zu schließen, indem zentrale Ergebnisse einer als Vollerhebung angelegten schriftlichen Befragung von Gesetzlichen Krankenkassen vorgestellt sowie anschließend diskutiert und bewertet werden.

\section{Hintergrund und Zielsetzung}

In den letzten Jahrzehnten sind die Ausgaben der Gesetzlichen Krankenversicherung $(\mathrm{GKV})$ rapide angestiegen. Zur Ausgabenbegrenzung bzw. zur Beeinflussung des Nachfrageverhaltens wurden von Seiten des Gesetzgebers verschiedene Reformmaßnahmen wie Leistungsausgrenzungen und Erhöhungen bzw. Einführungen von Zuzahlungen initiiert. Da die Erhebung von Zuzahlungen für Geringverdiener bzw. Versicherte mit einem schlechten Gesundheitszustand und demzufolge hohen Leistungsbedarf sehr schnell zu einer übermäßigen Belastung führen kann, wurden beginnend mit dem Gesundheitsreformgesetz (GRG) im Jahr 1989 Zuzahlungsbefreiungen - so genannte Härtefallregelungen - gesetzlich verankert. ${ }^{1}$ Die Definition eines Versicherten als Härtefall wird im Sozialgesetzbuch V (SGB V) verbindlich geregelt, wobei zunächst drei Befreiungstatbestände unterschieden wurden: ${ }^{2}$

- Versicherte, deren Bruttoeinnahmen zum Lebensunterhalt eine bestimmte Belastungsgrenze unterschreiten, sind von Zuzahlungen vollständig befreit ( $\int 61$ SGB V alt - Sozialklausel),

1 Vor 1989 lag die Anwendung der Härtefallregelungen, deren Ursprung im Krankenversicherungs-Kostendämpfungsgesetz (KVKG) von 1977 verankert ist, im Ermessen der jeweiligen Krankenkassen im Rahmen des Satzungsrechts.

2 Die abweichenden Regelungen für den Zahnersatz sind in § 55 SGB V gebündelt. 
Abbildung 1: Anzahl der Härtefälle nach § 62 SGB V nach Befreiungstatbestand 2004-2011, Angaben absolut in Mio.*

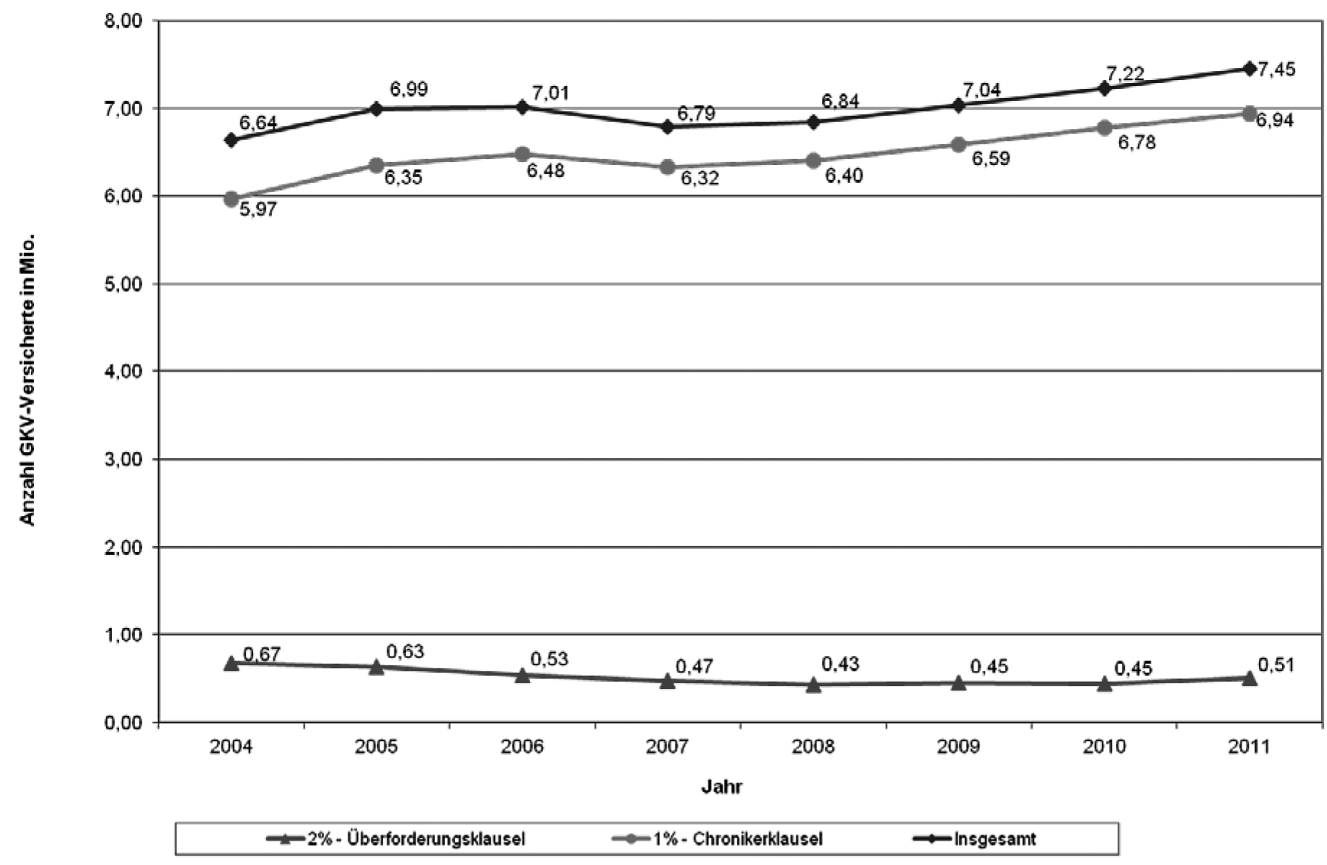

* eigene Darstellung auf Basis GBE (o. J.(a)).

bspw. im Jahr 2011 ca. $93 \%$ aller Härtefälle nach \62 SGB V aufgrund der Chronikerklausel von $\mathrm{Zu}$ zahlungen befreit waren (vgl. Abbildung 1).

Der zu beobachtende Anstieg der Härtefälle nach der Chronikerklausel in den Jahren 2004-2011 dürfte auf eine zunehmend ältere und damit multimorbidere Bevölkerung, die häufig unter die Chronikerklausel fällt, zurückzuführen sein. Der leichte Rückgang der Härtefallzahlen im Jahr 2007 deutet auf einen $\mathrm{Zu}$ sammenhang mit der im selben Jahr erfolgten Verschärfung der „Chroniker-Richtlinie“ durch den Gemeinsamen Bundesausschuss (G-BA) hin. Demgegenüber hat die schon im Jahr 2004 zahlenmäßig wesentlich unbedeutendere

- Versicherte, deren Zuzahlungen 2\% ihrer Bruttoeinnahmen zum Lebensunterhalt überschreiten, sind von weiteren Zuzahlungen befreit $(\mathbb{S} 62$ SGB V - Überforderungsklausel),

- Chronisch kranke Versicherte, deren Zuzahlungen 1\% ihrer Bruttoeinnahmen zum Lebensunterhalt überschreiten, sind von weiteren Zuzahlungen befreit $(\mathbb{S} 62$ SGB V Chronikerklausel).

Im Zuge des Gesundheitssystemmodernisierungsgesetzes (GMG) kam es ab dem Jahr 2004 zu grundlegenden Änderungen an den gesetzlichen Grundlagen der Härtefallregelungen, wobei an vorderster Stelle die Abschaffung der Sozialklausel zu nennen wäre (vgl. Langer (2005), S. 283 ff.). Zuletzt wurde im Gesetz zur Stärkung des Wettbewerbs in der Gesetzlichen Krankenversicherung (GKV-WSG) ab dem Jahr 2007 die Inanspruchnahme der Chronikerklausel an die Erfüllung weiterer Voraussetzungen ${ }^{3}$ geknüpft.

Neben den Zuzahlungsbefreiungen durch die „klassischen“ Härtefallregelungen des $\mathbb{} 62$ bzw. des $\mathbb{S} 55$ SGB V sind weitere Zuzahlungsbefreiungen für Minderjährige (mit Ausnahme des Zahnersatzes sowie der Fahrkosten) vorgesehen. Krankenkassen können darüber hinaus Zuzahlungen für Arz- neimittel um die Hälfte ermäßigen bzw. ganz aufheben, wenn für diese Arzneimittel eine bestimmte Rabattvereinbarung mit einem pharmazeutischen Unternehmen besteht und daraus Einsparungen zu erwarten sind. Zudem sind Zuzahlungsbefreiungen bei der Wahl bestimmter, preiswerter Arzneimittel sowie die Möglichkeit von Zuzahlungsreduktionen bei der Teilnahme an besonderen Versorgungsformen (bspw. Integrierte Versorgung) gesetzlich verankert. $^{4}$

Aufgrund des Umfangs der zu leistenden Zuzahlungen ${ }^{5}$ ist auch die Anzahl der von Härtefallregelungen profitierenden Versicherten entsprechend hoch. So lag der Anteil der Härtefälle an allen GKV-Versicherten im Jahr 2011 bei fast $11 \%$. Betrachtet man die Entwicklung der Härtefälle seit Inkrafttreten des GMG zum 01.01.2004 im zeitlichen Trend ${ }^{6}$, war in den Folgejahren 2005 und 2006 ein Anstieg der Anzahl der Härtefälle bis auf etwa 7 Mio. zu verzeichnen. 2007 kam es hingegen zu einem leichten Rückgang auf 6,79 Mio. Härtefalle. Im Zeitraum von 2008 bis 2011 war wiederum ein kontinuierlicher Anstieg um 0,61 Mio. auf 7,45 Mio. zu beobachten. Dabei wurde dieser Trend ganz maßgeblich von der Anzahl der Härtefälle nach der Chronikerklausel beeinflusst, da
Gruppe der Härtefälle nach der Überforderungsklausel im Zeitverlauf weiter leicht abgenommen. Ursächlich dafür dürfte - maßgeblich beeinflusst durch sinkende Arzneimittelzuzahlungen aufgrund diverser Arzneimittelsparmaßnahmen (vgl. Coca, Schröder (2012), S. 210 ff.) - das im selben Zeitraum rückläufige GKV-Zuzahlungsvolumen sein (vgl. GBE (o. J. (b))).

Der Bereich der Härtefallregelungen nach $\$ 62$ bzw. $\mathbb{5} 55$ SGB V war bislang äußerst selten Gegenstand wissenschaftlicher Forschungsvorhaben und -analysen bzw. entsprechender Veröffentli-

3 Siehe $§ 62$ SGB V in Verbindung mit der "Chroniker-Richtlinie" (vgl. G-BA (2008)) des Gemeinsamen Bundesausschusses (G-BA).

4 Bei der Überforderungsregelung des § 242b SGB V zur Vermeidung von Härten im Zusammenhang mit der Erhebung eines $\mathrm{Zu}$ satzbeitrags handelt es sich zwar ebenfalls um eine „Härtefallregelung“, allerdings steht diese in einem anderen inhaltlichen Zusammenhang als die Härtefallregelungen der $\$ \S 62$ bzw. 55 SGB V.

5 So lag das von GKV-Versicherten zu leistende Zuzahlungsvolumen im Jahr 2011 bei insgesamt 5,25 Mrd. EUR (vgl. GBE (o.J.(b))). Hinzu kommen weitere Belastungen durch Aufzahlungen und direkte Käufe, die nicht durch Härtefallregelungen abgefedert werden.

6 Die Zahlen vor 2004 sind aufgrund anderer gesetzlicher Regelungen nicht vergleichbar, daher wird hier auf die Entwicklung ab 2004 abgestellt (zu entsprechenden Zeitreihen vor 2004 vgl. Langer (2005), S. 163 ff.). 
chungen. ${ }^{7}$ Die wenigen Untersuchungen ließen bisher die Sichtweise der Gesetzlichen Krankenkassen im Hinblick auf die administrative Umsetzung der Härtefallregelungen bzw. deren mögliches Optimierungspotential weitgehend unberücksichtigt. Diese gewinnt gerade vor dem Hintergrund der ständigen Diskussion über die angemessene Höhe der GKV-Verwaltungsausgaben an Relevanz, da Härtefallregelungen einen nicht unerheblichen Verwaltungsaufwand und damit entsprechende Ausgaben auf Seiten der Krankenkassen verursachen.

Im Rahmen der empirischen Erhebung standen daher folgende Fragestellungen im Vordergrund:

- Wie werden die Versicherten hinsichtlich der Existenz der Härtefallregelungen informiert? Wie aus einer früheren Untersuchung hervorgeht, sind vielen Versicherten die Härtefallregelungen nicht bekannt (vgl. Eller, Baumann, Mielck (2002), S. 569 ff.). Aufgrund der finanziellen Belastungen durch die Härtefallregelungen und der vermutlich nur unzureichenden Refinanzierung haben die Krankenkassen einen Anreiz, Versicherte über die Existenz dieser Normen evt. nicht in Kenntnis zu setzen. Dadurch würde die Effektivität des Instruments „Härtefallregelungen“ deutlich eingeschränkt werden.

- Wie erfolgt die organisatorische Umsetzung der Härtefallregelungen durch die Gesetzlichen Krankenkassen? Zum einen stellt sich die Frage, ob die Krankenkassen aus Gründen der Verwaltungsökonomie eher eine zentrale oder dezentrale Bearbeitung präferieren. Zum anderen ist interessant, welche Prüftiefe die Krankenkassen nach Art und Umfang der einzureichenden Unterlagen für notwendig halten.

- Gibt es Alternativen zum Antragsverfahren und wie werden diese beurteilt? Im aktuellen Antragsverfahren ist nicht auszuschließen, dass manche GKV-Versicherte keinen Befreiungsantrag gestellt haben, obwohl sie bei erfolgter Antragstellung unter die Härtefallregelung gefallen wären (sog. „Dunkelziffer“). Wie beurteilen die Krankenkassen daher alternative Methoden, insbesondere ein „automatisiertes" Verfahren, hinsichtlich des trade-offs aus Beachtung der Verwaltungskosten und der Realisie-

Tabelle 1: Verteilung der Krankenkassen nach Kassenarten laut GKV- Spitzenverband sowie in der Datengrundlage, Angaben absolut und in \%*

\begin{tabular}{|l|c|c|c|}
\hline Kassenart & $\begin{array}{c}\text { Anzahl Krankenkassen } \\
\text { laut GKV-SV } \\
\text { am 01.10.2012 }\end{array}$ & $\begin{array}{c}\text { Anzahl Krankenkassen } \\
\text { in der Datengrundlage }\end{array}$ & $\begin{array}{c}\text { Anteil Krankenkassen } \\
\text { in der Datengrundlage } \\
\text { in \% }\end{array}$ \\
\hline AOK & 11 & 9 & 81,8 \\
\hline VdEK & 6 & 5 & 83,3 \\
\hline BKK & 110 & 55 & 50,0 \\
\hline IKK & 6 & 3 & 50,0 \\
\hline LKK & 9 & 8 & 88,8 \\
\hline Knappschaft & 1 & 0 & 0 \\
\hline Gesamt & 143 & 80 & 55,9 \\
\hline
\end{tabular}

* GKV-SV (o. J.(a)) sowie eigene Erhebung.

rung möglichst umfassender sozialer Gerechtigkeit?

- Wird der Wettbewerb unter den Krankenkassen durch die Härtefallregelungen beeinflusst? Die Einführung der Krankenkassenwahlfreiheit im Jahr 1996 löste einen intensiven (Preis-)Wettbewerb um Versicherte zwischen den Krankenkassen aus. Vor diesem Hintergrund stellt sich daher die Frage, ob und in welcher Form die Härtefallregelungen einen Einfluss auf das Wettbewerbsgeschehen in der Gesetzlichen Krankenversicherung haben.

- Welche Optimierungsmöglichkeiten gibt es hinsichtlich der Administration der Härtefälle?

\section{Studiendesign und Datengrundlage}

\subsection{Studiendesign}

Zur Umsetzung der Studie wurde zunächst auf die Ergebnisse einer Vorstudie zurückgegriffen. Diese erfolgte im Rahmen von Leitfadeninterviews, anhand derer wichtige administrative Aspekte ermittelt wurden. Dabei fand eine Befragung von acht Härtefallsachbearbeitern unterschiedlicher Gesetzlicher Krankenkassen statt. Darauf aufbauend wurde ein Fragebogen konstruiert, der neben statistischen Angaben über die teilnehmenden Gesetzlichen Krankenkassen elf sowohl geschlossene als auch offene Fragen beinhaltete. Die geschlossenen Fragen waren anhand zwei- bis fünfstufiger Skalen zu beantworten. Die Analyse der gewonnenen Daten erfolgte vorrangig mittels deskriptiver Statistik. An einigen Stellen wurden die Ergebnisse um analytische statistische Verfahren ergänzt. Für die
Erfassung und Analyse der Daten wurde die Statistiksoftware SPSS 20 eingesetzt.

\subsection{Datengrundlage}

Von März bis April 2012 wurde ein Fragebogen konstruiert, der im Mai 2012 insgesamt vier Gesetzlichen Krankenkassen bzw. den jeweiligen Fachverantwortlichen im Rahmen eines Pretests ${ }^{8}$ vorgelegt wurde. Die dabei gewonnenen Erkenntnisse wurden Ende Mai in den endgültigen Fragebogen eingearbeitet. Zur Erhöhung der Rücklaufquote wurden im Juni 2012 alle Gesetzlichen Krankenkassen telefonisch mit dem Ziel kontaktiert, einen fachlich geeigneten Ansprechpartner für die Beantwortung des Fragebogens zu ermitteln. Im Juli 2012 wurden alle 143 Gesetzlichen Krankenkassen in Deutschland (Stand 01.06.2012) bzw. die jeweils ermittelten Ansprechpartner postalisch angeschrieben. Sie wurden gebeten, den ausgefüllten Fragebogen im beigelegten, bereits frankierten Rückumschlag zurückzusenden. Die Untersuchung ist somit als Vollerhebung angelegt. Nach einem ersten Rücklauf erfolgte zur Erhöhung der Antwortrate im September 2012 zunächst eine schriftliche (mit der nochmaligen Übersendung des Fragebogens inkl. Rückumschlag) sowie im

7 Die Ergebnisse werden beschrieben in Pfaff et al. (2002); Eller, Baumann, Mielck (2002); Langer et al. (2003); Eller et al. (2004); Langer (2005); Langer, Pfaff, Kern (2006).

8 Der Pretest erfolgte mit vier Fachverantwortlichen in der Region Neubrandenburg (regionale Ebene), wohingegen sich die schriftliche Befragung derselben Krankenkassen an die jeweiligen Härtefallverantwortlichen auf Bundesebene richtete. Insofern kann eine doppelte Befragung derselben Probanden (Pretest und Hauptbefragung) ausgeschlossen werden. 
Tabelle 2: Verteilung der Versicherten nach Kassenarten in der Statistik KM6 sowie in der Datengrundlage, Angaben absolut und in \%*

\begin{tabular}{|l|r|r|r|r|c|}
\hline Kassenart & $\begin{array}{c}\text { Anzahl Versicherte } \\
\text { (Statistik KM6 zum } \\
\text { 01.07.2012) }\end{array}$ & $\begin{array}{c}\text { Anzahl Versicherte } \\
\text { (Datengrundlage) }\end{array}$ & $\begin{array}{c}\text { Versicherte in der Daten- } \\
\text { grundlage als Anteil an allen } \\
\text { Versicherten nach Statistik } \\
\text { KM6 zum 01.07.2012 }\end{array}$ & $\begin{array}{c}\text { Anteil Versicherte } \\
\text { in \% } \\
\text { (Statistik KM6 zum } \\
\text { 01.07.2012) }\end{array}$ & $\begin{array}{c}\text { Anteil Versicherte } \\
\text { in \% } \\
\text { (Datengrundlage) }\end{array}$ \\
\hline AOK & 24.343 .809 & 21.320 .000 & 87,6 & 34,9 & 37,2 \\
\hline VdEK & 25.729 .735 & 24.556 .000 & 95,4 & 36,9 & 42,9 \\
\hline BKK & 11.657 .595 & 6.127 .995 & 52,6 & 16,7 & 10,7 \\
\hline IKK & 5.463 .758 & 4.526 .631 & 82,9 & 7,8 & 7,9 \\
\hline LKK & 766.544 & 743.743 & 97,0 & 1,2 & 1,3 \\
\hline Knappschaft & 1.754 .775 & 0 & 0 & 2,5 & 0 \\
\hline Gesamt & 69.716 .216 & 57.274 .369 & 82,1 & 100 & 100 \\
\hline
\end{tabular}

* BMG (o. J.) sowie eigene Erhebung

Oktober 2012 eine zusätzliche telefonische Nachfassaktion (mit der Bitte, den bereits schriftlich übersendeten Fragebogen postalisch zurückzusenden).

Durch dieses mehrstufige Vorgehen konnte schließlich ein Rücklauf von 80 Fragebögen erzielt werden, sodass die Rücklaufquote auf Krankenkassenebene bei ca. 56\% lag. ${ }^{9}$ Alle Fragebögen wurden in die Analyse einbezogen, auch wenn von manchen Teilnehmern einzelne Fragen nicht beantwortet bzw. ungültige Mehrfachantworten gegeben wurden. Mit Ausnahme der Knappschaft, die nicht an der Befragung teilnahm, lagen die kassenbezogenen Rücklaufquoten bei allen Kassenarten zwischen 50\% und ca. 89\% (vgl. Tabelle 1). Die Quote von $50 \%$ bei den Betriebskrankenkassen

\section{Abbildung 2: Information der Versicherten hinsichtlich der Existenz der Härtefall- regelungen*}

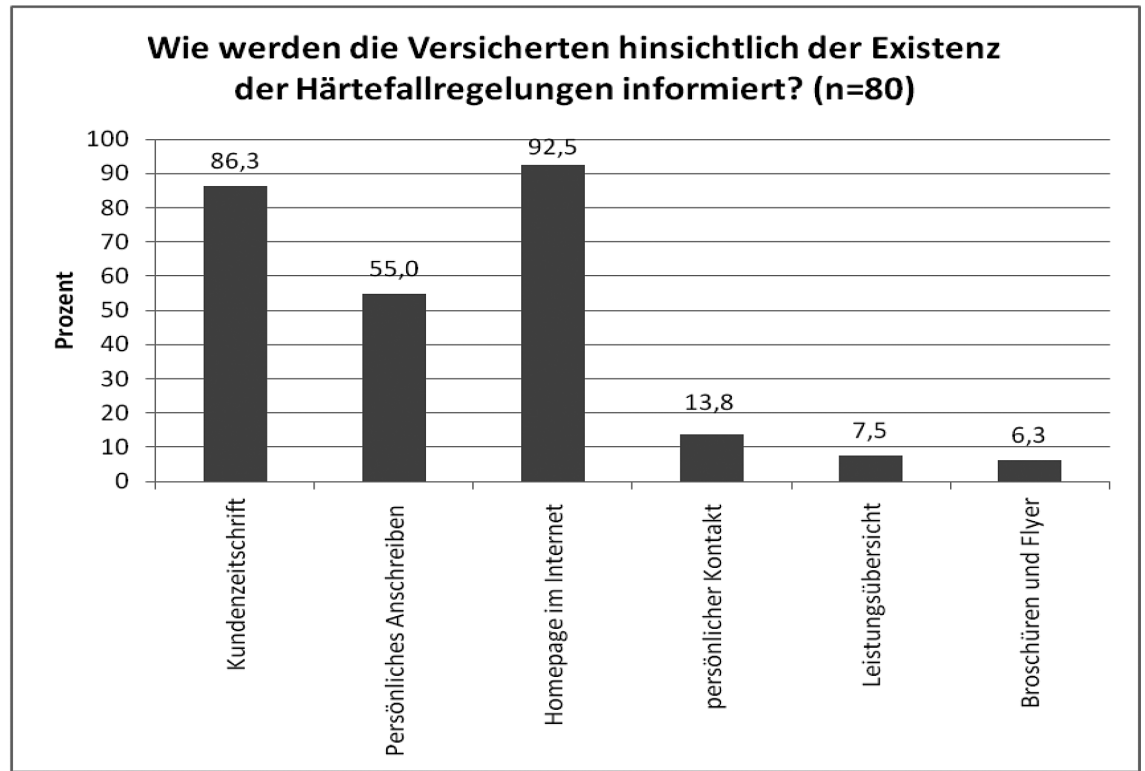

* eigene Darstellung; Da Mehrfachnennungen möglich waren, ergeben die einzelnen Balken in der Summe mehr als $100 \%$.
(BKK) ist dabei als zufriedenstellend $\mathrm{zu}$ bewerten, wenn man die geringe Versichertenzahl einiger BKKn und die damit einhergehenden geringen Kapazitäten zur Beantwortung der Fragebögen bedenkt (vgl. SVRG (2012), S. 342).

Betrachtet man hingegen den Anteil der Versicherten in der Datengrundlage, so repräsentieren die 80 antwortenden Krankenkassen sogar ca. 82\% der Versicherten (ca. 57,3 Mio. Versicherte) (vgl. Tabelle 2). Trotz der Nichtteilnahme von drei Innungskrankenkassen (IKK) liegt der versichertenbezogene Anteil der IKKn bei ca. 83\%. Der Großteil der Versicherten der Innungskrankenkassen wird dementsprechend mit der vorliegenden Studie repräsentiert. Zudem verdeutlicht Tabelle 2 , dass vor allem die AOKen sowie die
Ersatzkassen (VdEK) bezogen auf die Versichertenzahl in der Datengrundlage überdurchschnittlich, die BKKen hingegen nur unterdurchschnittlich vertreten sind.

\section{Ergebnisse}

Die Krankenkassen wurden gebeten, anzugeben, in welcher Form sie ihre Versicherten hinsichtlich bestehender Härtefallregelungen informieren (vgl. Abbildung 2). Dabei zeigt sich, dass das am meisten genutzte Medium zur Information der Versicherten die Homepage im Internet darstellt (92,5\%), gefolgt von der Kundenzeitschrift (86,3\%) sowie einem persönlichen Anschreiben $(55,0 \%)$. Als weitere Informationskanäle werden von den Krankenkassen Angebote wie der persönliche Kontakt (13,8\%), eine Leistungsübersicht $(7,5 \%)$ sowie Broschüren und Flyer $(6,3 \%)$ genannt, um auf die Härtefallregelungen aufmerksam zu machen. Zum persönlichen Kontakt zählen persönliche oder telefonische Beratungen sowie der Versand von E-Mails. In einer Leistungsübersicht sind die einzelnen Leistungen der Krankenkasse aufgelistet, die den Versicherten angeboten werden (Maßnahmen, Programme, Beratungen, etc.).

Die Informationsübermittlung auf der Homepage stellt für eine Krankenkasse eine attraktive Möglichkeit der Versicherteninformation dar, da einerseits die Informationen ständig abrufbar sind und andererseits die administrativen Umsetzungskosten vergleichsweise gering ausfallen. Daher ist es nachvollziehbar, dass

9 Zum Vergleich: Die Rücklaufquoten von aktuelleren Krankenkassenbefragungen anderer Autoren lagen bspw. bei ca. 29\% (vgl. Krummaker et al. (2008)) bzw. bei 69\% (vgl. SVRG (2012)). 


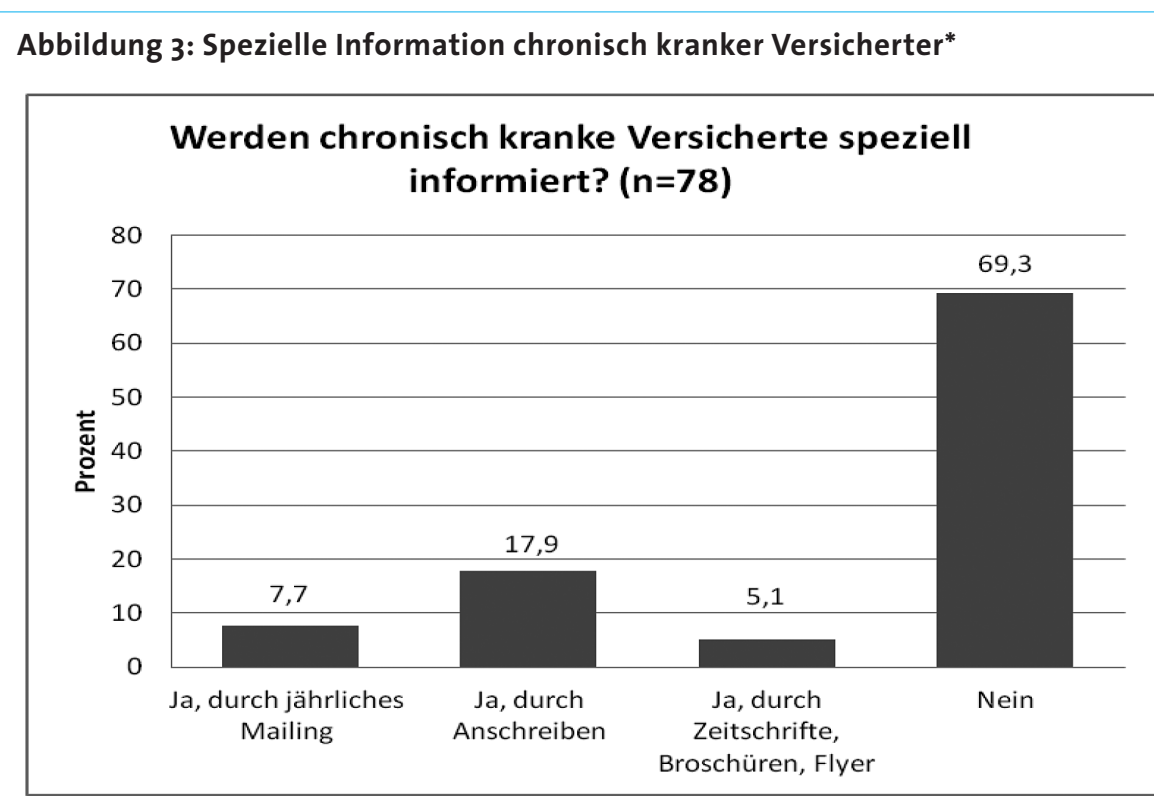

* eigene Darstellung; Aufgrund fehlender Angaben wurden 78 Krankenkassen berücksichtigt.

die Mehrheit der Kassen diesen Weg nutzt, um ihre Versicherten zu informieren. Da es sich bei den Härtefallregelungen um ein Antragsverfahren handelt, sollte jedoch unter sozialpolitischen Aspekten beachtet werden, welche der genannten Informationskanäle die größte Aufmerksamkeit bei den Versicherten erlangen. Eine Homepage muss aus eigener Initiative aufgerufen werden, um Informationen einzuholen, wohingegen eine Kundenzeitschrift eine direkte Informationsübermittlung an den Empfänger initiiert. Ähnlich vorteilhaft ist der postalische Weg, der trotz entsprechendem finanziellen Aufwands von einem Großteil der Krankenkassen genutzt wird. Den nachhaltigsten Eindruck hinterlässt sicherlich ein persönliches Gespräch bzw. ein Telefonat. Beide Optionen, die den Versicherten gleichzeitig die beste Möglichkeit bieten, bestehende Fragen zu beantworten, stellen für die Krankenkassen allerdings einen hohen Verwaltungsaufwand dar. Dies könnte ein Grund dafür sein, dass relativ wenige der befragten Krankenkassen diese Art der Auskunft zur Information ihrer Versicherten nutzen.

Hinsichtlich der Erreichbarkeit ist zudem darauf zu achten, dass die Adressaten in der Lage sind, die Informationen zu empfangen. So kann davon ausgegangen werden, dass eine Homepage überwiegend von jüngeren Versicherten abgerufen wird.

Die Frage nach der speziellen Information chronisch kranker Versicherter beantworteten 78 der befragten Krankenkassen (vgl. Abbildung 3). Knapp

lung als nicht notwendig erachtet wird.

\section{Abbildung 4: Bearbeitung der Anträge*} berücksichtigt.
Die Organisation eines Unternehmens kann vielfältig gestaltet werden und hängt von verschiedenen Faktoren ab. Dabei gibt es jedoch keine bestimmten idealtypischen Vorgaben oder Vorschriften, vielmehr ist die Form der Organisationsstruktur von der individuellen Situation abhängig. Der Grad der Zentralisierung oder Dezentralisierung stellt eines der wichtigsten Kriterien der Organisationsstruktur dar. Zentralisation wird als die Konzentration merkmalsgleicher Teilaufgaben in einem Zentrum charakterisiert (vgl. Frese (2005), S. 233). Organisationen können sich auf diese Art stärker spezialisieren. Bei dezentralen Entscheidungen hingegen wird Raum für lokale Anpassungen gelassen (vgl. Kieser, Walgenbach (2010), S. 282) und merkmalsgleiche Teilaufgaben über mehrere Einheiten parallel verteilt (vgl. Frese (2005), S. 233). Die Organisation kann durch dezentrale Aufgabenverteilung i.d.R. kundenorientierter handeln. Dies lässt jedoch weniger Raum für Spezialisierungen.

Um Anträge von Versicherten mit Härtefallstatus zu bearbeiten, bestehen nun die genannten Möglichkeiten der zentralen und dezentralen Bearbeitung sowie die Kombination beider Formen. Wie aus Abbildung 4 hervorgeht, nutzen ca. zwei Drittel der befragten Krankenkassen die dezentrale Bearbeitung durch die jeweils zuständigen Sachbearbeiter $(66,2 \%)$. Damit wird die dezentrale Bearbeitung im Vergleich mit der zentralen Bearbeitung $(18,9 \%)$ von den befragten Kran-

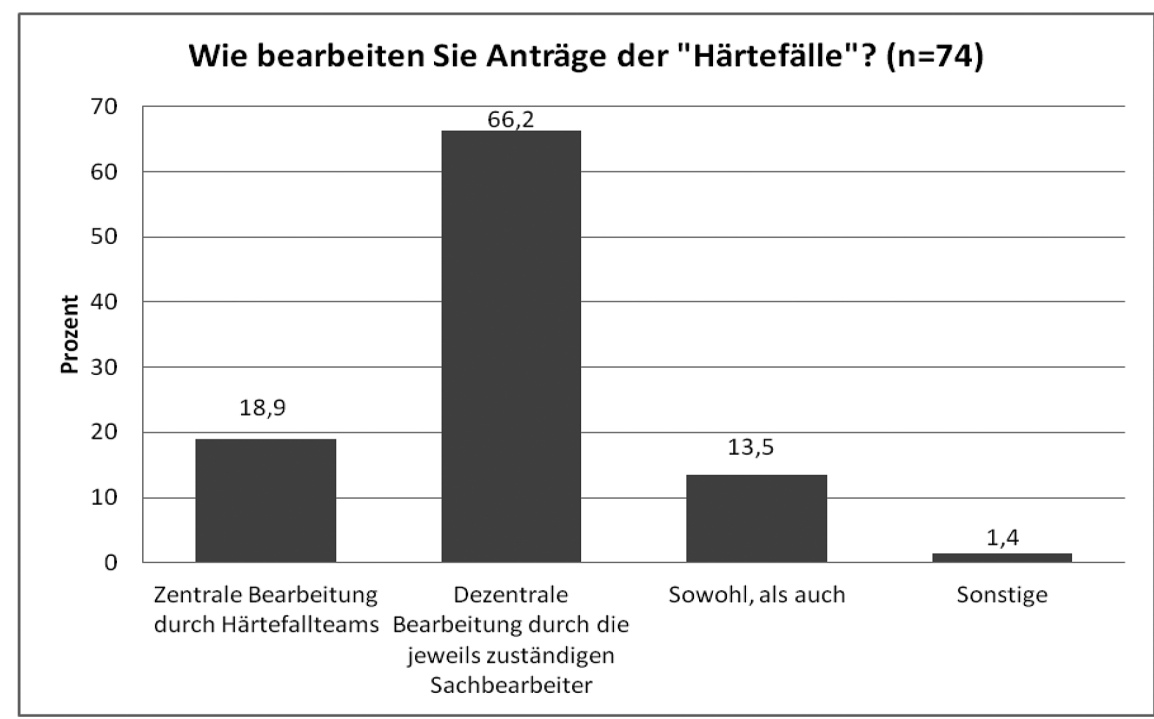

* eigene Darstellung; Aufgrund ungültiger Mehrfachnennungen wurden 74 Krankenkassen 
kenkassen mehr als dreimal so häufig praktiziert. $\mathrm{Zu}$ „Sonstige“ zählt hier bspw. eine zentrale Bearbeitung durch Leiharbeitnehmer in Zusammenarbeit mit eigenen Mitarbeitern.

Da die Wahl der Aufgabenverteilung bei jeder Organisation nicht nur von der Unternehmensgröße, sondern auch von anderen Einflussfaktoren wie finanziellen Ressourcen oder kulturellen Faktoren abhängt, wurde von den befragten Krankenkassen im Sinne des „situativen Ansatzes“ eine geeignete Form der Antragsbearbeitung gewählt. Die Ergebnisse der Umfrage lassen daher auch keinen signifikanten Zusammenhang zwischen der Größe einer Krankenkasse und dem bevorzugten Zentralisierungsgrad erkennen.

Wie Abbildung 5 zeigt, bieten alle antwortenden Krankenkassen Möglichkeiten der Vorabbefreiung. Es ist ersichtlich, dass mehr als die Hälfte der Krankenkassen $(56,8 \%)$ eine solche Vorabbefreiung nur für spezifische Gruppen ${ }^{10}$ vorsehen. Demgegenüber stehen $43,2 \%$ der Krankenkassen, die an dieser Stelle keine Ausnahmen machen und grundsätzlichen allen Versicherten eine Vorabbefreiung ermöglichen. In diesem Zusammenhang hat sich gezeigt, dass kleine Krankenkassen signifikant häufiger als große Krankenkassen allen Versicherten Vorabbefreiungen bieten $\left(\chi^{2}[\mathrm{df}=1, \mathrm{n}=74]=4,763 ; \mathrm{p}<0,05\right) .{ }^{11}$

Vorabbefreiungen erfolgen vor allem aus Gründen der Verwaltungsökonomie. Durch das Angebot dieser Mög-

\section{Abbildung 5: Möglichkeiten der Vorabbefreiung*}

Bieten Sie Möglichkeiten der Vorabbefreiung? $(n=74)$

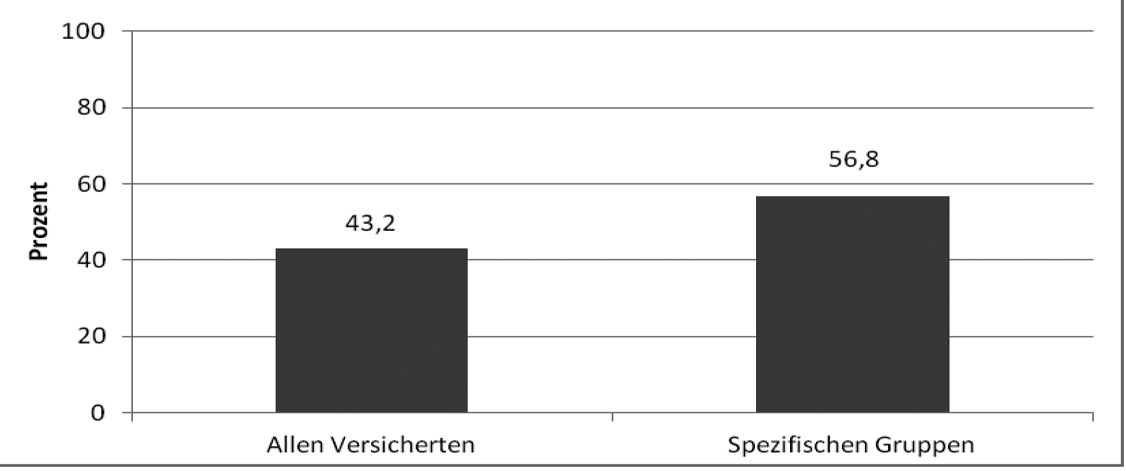

* eigene Darstellung; Aufgrund ungültiger Mehrfachnennungen wurden 74 Krankenkassen berücksichtigt. lichkeit kann der Verwaltungsaufwand aufgrund der Vermeidung arbeitsintensiver Prüfungen (vgl. Deutscher Bundestag (2006), S. 2) erheblich reduziert und somit Kosten eingespart werden. Zudem entfällt bei der Vorabbefreiung „das Sammeln und Einreichen der $\mathrm{Zu}$ zahlungsquittungen" (Deutscher Bundestag (2006), S. 2). Insbesondere bei spezifischen Versichertengruppen, wie z.B. Rentnern und Sozialhilfeempfängern, bietet sich dieses Vorgehen an, weil sich deren Bruttoeinnahmen zum Lebensunterhalt bzw. Chronikerstatus über einen längeren Zeitraum aller Wahrscheinlichkeit nach nicht ändern.

Die administrative Umsetzung der Vorabbefreiung erfolgt nach den Verfah-
Abbildung 6: Prüfung der Zuzahlungsbelege bei Antragsstellung*



rensgrundsätzen zu $\mathbb{6} 62$ SGB V. Ist ein Versicherter davon überzeugt, dass er im kommenden Kalenderjahr die Belastungsgrenze innerhalb eines kurzen Zeitraums erreicht, kann er bereits am Ende des aktuellen Kalenderjahres einen Antrag auf Zuzahlungsbefreiung stellen. Die Krankenkasse errechnet dann anhand der voraussichtlichen Bruttoeinnahmen zum Lebensunterhalt die individuelle Belastungsgrenze. Der so ermittelte $\mathrm{Zu}$ zahlungsbetrag ist dann vom Versicherten an die Krankenkasse zu überweisen. Anschließend erhält dieser zum Jahresbeginn den Befreiungsausweis. So kann sichergestellt werden, dass der Versicherte keine Zuzahlungen mehr leisten muss und auch auf das aufwändige Sammeln der Belege verzichten kann. Sollte die Belastungsgrenze jedoch nicht erreicht werden, besteht keine Möglichkeit der auch nur teilweisen Rückerstattung des vorab geleisteten Zuzahlungsbetrags. In Fällen, in denen die erwarteten Bruttoeinnahmen zum Lebensunterhalt nicht erreicht wurden, kann die Belastungsgrenze auf Antrag durch die Krankenkasse allerdings erneut ermittelt werden (vgl. GKV-SV (2010), S. 6 ff.).

Aus Abbildung 6 geht hervor, dass der Großteil (93,3\%) der Krankenkassen den Namen und das Ausstellungsdatum auf der Quittung bei der Prüfung der Zuzahlungsbelege als zwingend notwendig erachtet, was den Verfahrensgrund-

10 z.B. Rentner, Sozialhilfeempfänger oder Versicherte mit festem Einkommen.

11 Die Krankenkassen wurden dazu in kleine ( $\leq 100.000$ Versicherte) und große Krankenkassen (> 100.000 Versicherte) unterteilt. 




* eigene Darstellung; Aufgrund ungültiger Mehrfachnennungen wurden 72 Krankenkassen berücksichtigt. sätzen zu $\int 62$ SGB V entspricht (vgl. GKV-SV (2010), S. 16). Einem geringen Teil von nur fünf Krankenkassen (6,7\%) befreiten auf der Quittung aus. Alle Krankenkassen gaben an, eine obligatorische Prüfung der Zuzahlungsbelege durchzuführen, so dass diese keinesfalls stichprobenartig erfolgt oder dem Erreicht allein der Name des Zuzahlungs- messen des Sachbearbeiters unterstellt wird.

Ein ähnliches Muster weist die Einkommensprüfung auf (vgl. Abbildung 7). Auch hier halten nahezu alle Krankenkassen $(98,6 \%)$ den Einkommensnachweis des Versicherten für zwingend erforderlich. Lediglich bei einer Krankenkasse (1,4\%) liegt die
Abbildung 8: Prüfung der Bescheinigung über schwerwiegende chronische Erkrankungen*

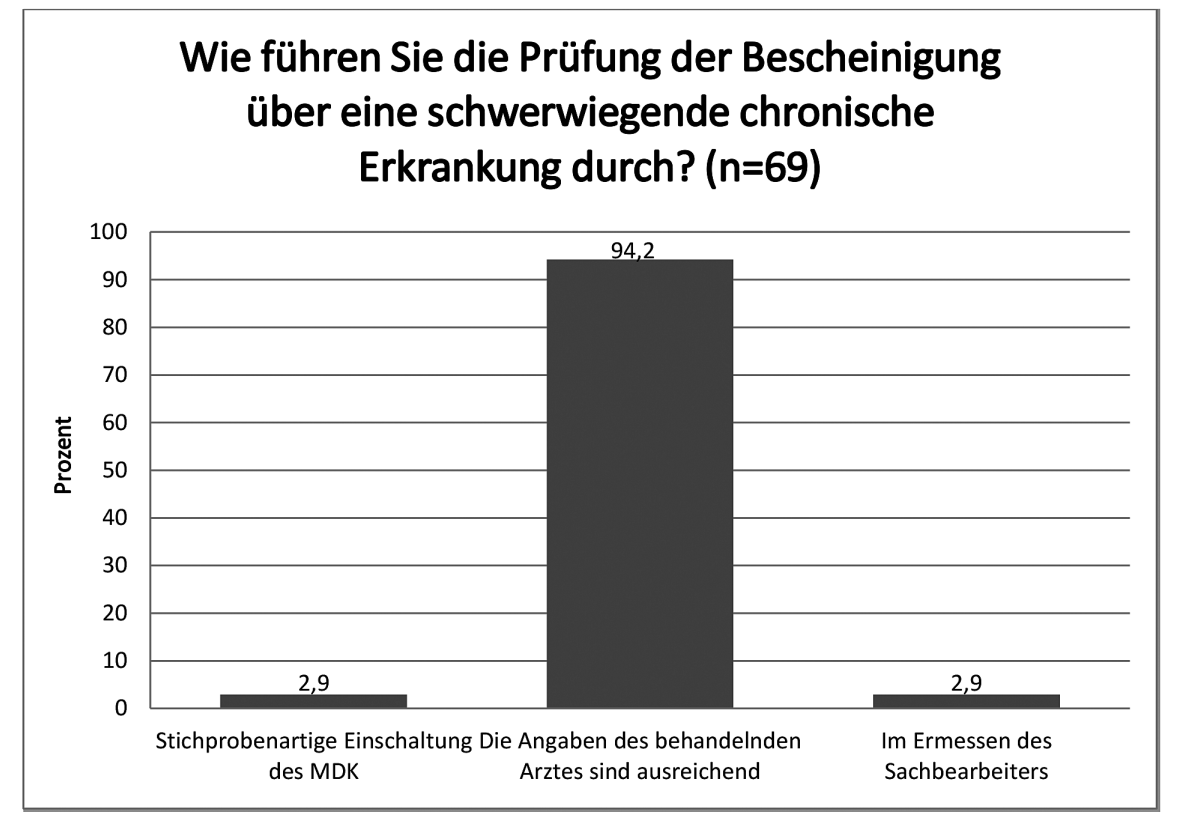

* eigene Darstellung; Aufgrund ungültiger Mehrfachnennungen wurden 69 Krankenkassen berücksichtigt.
Einkommensprüfung im Ermessen des Sachbearbeiters. Insgesamt zeigt sich bei der Zuzahlungs- und Einkommensprüfung eine intensive Prüftiefe, die dementsprechend auch zu hohen administrativen Kosten führt. Andererseits ist ohne eine exakte Prüfung der Zuzahlungsbzw. Einkommenssituation der Antragsteller auch nicht valide ermittelbar, ob ein Versicherter als zuzahlungsbefreit einzustufen ist.

Hingegen weist die Vorgehensweise der Krankenkassen bei der Bescheinigungsprüfung chronischer Krankheiten eine geringere Prüftiefe auf, wie Abbildung 8 zeigt.

Für nahezu alle Krankenkassen (94,2\%) sind hierbei die Angaben des behandelnden Arztes ausreichend. Die Einschaltung des MDK wird hingegen nur äußerst selten veranlasst $(2,9 \%)$. Dies hätte zwar grundsätzlich den Vorteil, die Kriterienprüfung außerhalb des Arzt-Patienten-Verhältnisses durchzuführen und damit gewissen Manipulationsgefahren aufgrund der „weichen“ gesetzlichen Regelungen vorzubeugen (vgl. Langer (2005), S. 325). Ein derartiges Vorgehen würde allerdings - nicht zuletzt auch aufgrund der hohen Fallzahlen - zu einem hohen Verwaltungsaufwand und damit zu hohen administrativen Kosten führen.

Wie aus Abbildung 9 hervorgeht, hält es eine deutliche Mehrheit der Krankenkassen für sinnvoll, am Antragsverfahren festzuhalten $(82,7 \%)$. Begründet wird diese Einschätzung zum einen mit den gesetzlichen Vorgaben (23,1\%). Etwa jede fünfte Kasse (19,2\%) ist zudem überzeugt, dass sich durch ein derartiges Verfahren Einkommensschwankungen besser ermitteln und nachvollziehen lassen. Etwa jede siebente Kasse (15,4\%) sieht im Antragsverfahren ein vereinfachtes Verwaltungsverfahren. Weiterhin begründet in etwa jede zehnte Kasse (11,5\%) die Sinnhaftigkeit des Antragsverfahrens mit der Gleichbehandlung aller Versicherten.

Der Großteil der Krankenkassen, die das Antragsverfahren ablehnen, begründen dies mit dem erhöhten Verwaltungsaufwand $(15,4 \%)$. Alternativ wird von diesen Krankenkassen ein „automatisiertes“ Verfahren - bei dem die Anspruchsberechtigung für jeden Versicherten ohne explizite Antragstellung geprüft wird - sozialpolitisch für sinnvoll erachtet. Da diese Krankenkas- 
Abbildung 9: Festhalten am Antragsverfahren*

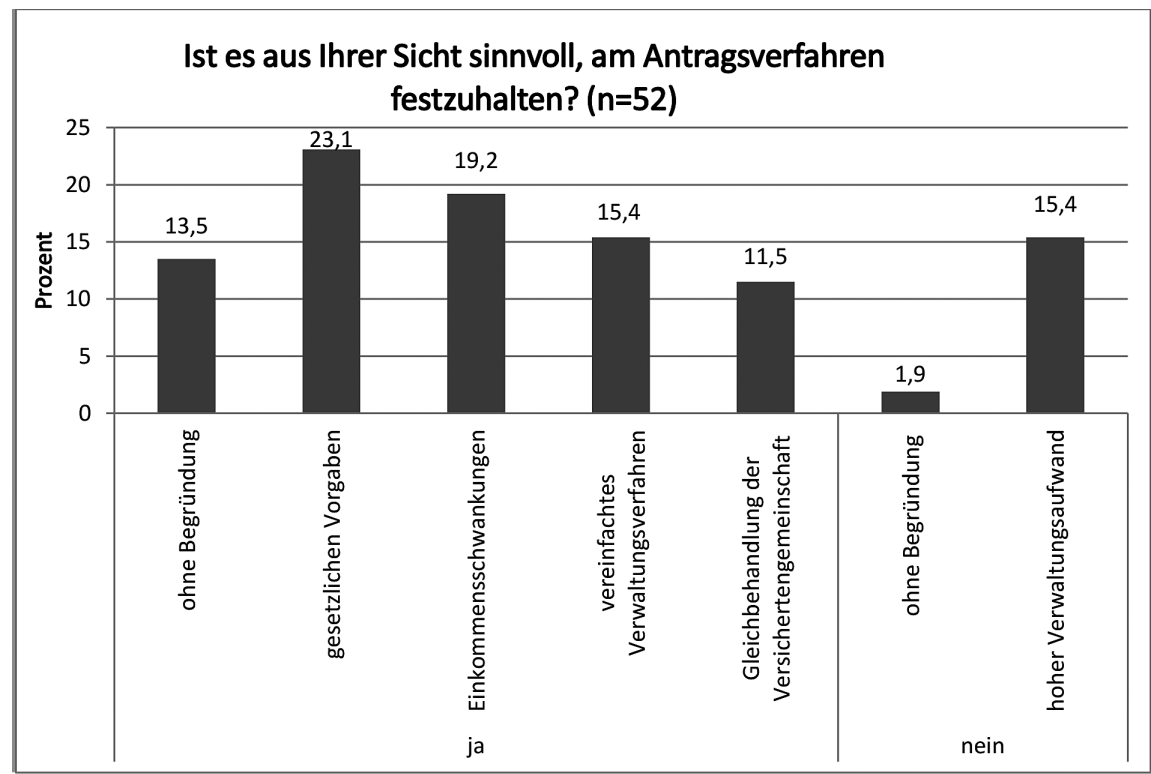

* eigene Darstellung; Aufgrund fehlender Angaben wurden 52 Krankenkassen berücksichtigt.

sen aber schon das bisherige Antragsverfahren aufgrund des hohen Verwaltungsaufwandes ablehnen, halten sie ein verwaltungsökonomisch noch aufwändigeres „automatisiertes“ Verfahren allerdings für nicht realisierbar.

Wie Abbildung 10 zeigt, sind nur 2 der antwortenden Krankenkassen (2,6\%) der Meinung, dass die Härtefallregelungen einen relevanten Einfluss auf das Wettbewerbsgeschehen in der Gesetzlichen Krankenversicherung haben. Wesentlich mehr Krankenkassen (20,8\%) sehen hingegen durch die Härtefallregelungen eine teilweise Beeinflussung des Wettbewerbs. Begründet wird diese An-

sicht zum einen mit der Möglichkeit der gezielten Anwerbung von guten Risiken anderer Krankenkassen, da bei der Beteiligung mehrerer Krankenkassen zur Umsetzung des Haushaltsbezugs Einkommens- und Gesundheitszustandsinformationen für die Fremdversicherten vorliegen. Zum anderen wird auch in der teilweise unterschiedlichen Umsetzung der gesetzlichen Regelungen eine Wettbewerbsbeeinflussung gesehen, so bspw. in den unterschiedlich großzügig gestalteten Regelungen zur Vorabbefreiung. Die überwiegende Anzahl der Krankenkassen $(76,6 \%)$ sieht den Wettbewerb hingegen durch die Här-

\section{Abbildung 10: Einfluss auf den Wettbewerb *}

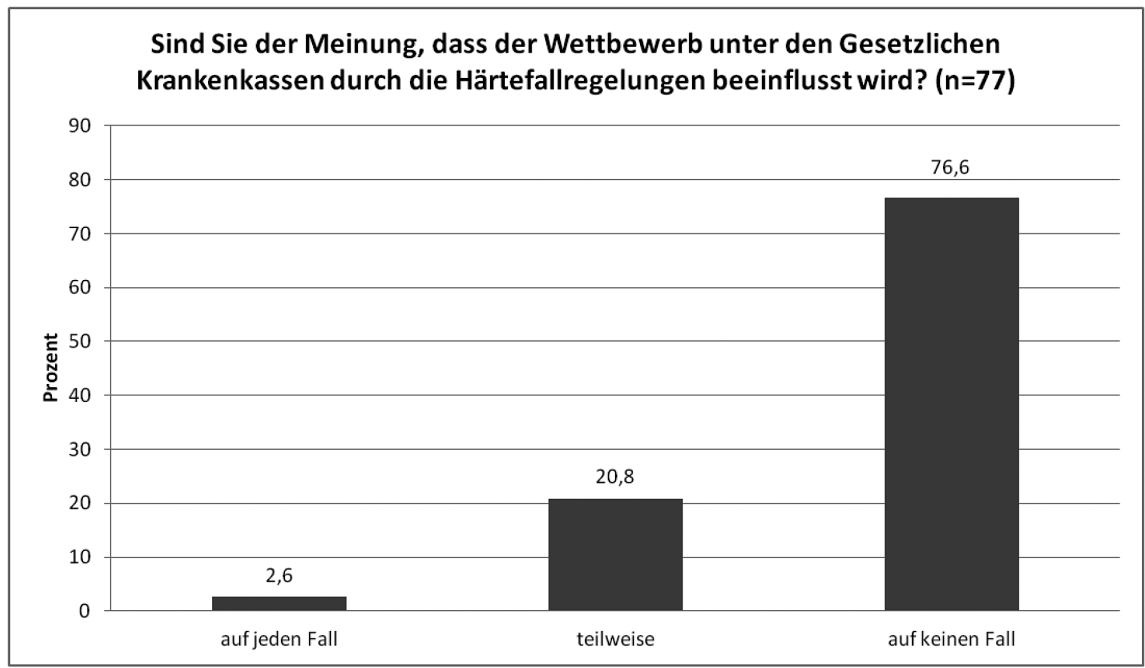

* eigene Darstellung; Aufgrund fehlender Angaben wurden 77 Krankenkassen berücksichtigt. tefallregelungen auf keinen Fall beeinflusst. Als Gründe hierfür werden vor allem die einheitlichen gesetzlichen Regelungen im SGB V genannt, die zudem durch entsprechende Rundschreiben und Verfahrensgrundsätze noch einheitlich konkretisiert werden, so dass kein Ermessensspielraum besteht. Außerdem werden die Härtefallbefreiten als eher schlechte Risiken gesehen, die darüber hinaus noch wenig Wechselbereitschaft zeigen, sodass der betroffene Personenkreis aus Wettbewerbs- bzw. Marketingsicht für die Krankenkassen nicht sonderlich interessant ist.

Rund $86 \%$ der antwortenden Krankenkassen ${ }^{12}$ sehen Optimierungsmöglichkeiten hinsichtlich der Administration der Härtefälle. Unter den genannten Möglichkeiten wurden zum einen unter dem Aspekt einer Reduzierung des hohen Verwaltungsaufwandes eine Abschaffung der Chronikerklausel und die generelle Festlegung einer einheitlichen Grenze ( $1 \%$ oder $2 \%$ ) für alle Härtefälle des Öfteren vorgeschlagen. Ein weiterer mehrfach genannter Aspekt zur Verwaltungsvereinfachung ist die geforderte Wiedereinführung einer festen Einkommensgrenze für bestimmte Personengruppen (bspw. Hartz IVEmpfänger) bzw. generell für Geringverdiener in Anlehnung an die Regelungen des $\$ 61$ SGB V in der Fassung bis Ende 2003. Darüber hinaus halten einige Krankenkassen die Abschaffung der ihrer Ansicht nach „unsinnigen Vorsorgeklausel bzw. Malusregelung“ des $\mathbb{6} 62$ SGB V für geboten. Im Sinne einer „automatisierten" Bearbeitung wünschen sich manche Krankenkassen zudem eine Software, die Zuzahlungs- und Einkommensinformationen systemseitig aus den vorhandenen Daten generieren kann.

\section{Diskussion}

Wie die Analyse gezeigt hat, geben die Krankenkassen an, vielfältige Informationskanäle zur Information ihrer Versicherten zu nutzen. Dabei werden nicht nur „neue Medien“ wie die Homepage eingesetzt, deren Nachteil vor allem darin liegt, dass derartige Informationswege von den älteren, von Härtefallregelungen besonders betroffenen Versicherten, bislang noch

12 Aufgrund fehlender Angaben wurden 42 Krankenkassen berücksichtigt. 
nicht ausreichend genutzt werden. ${ }^{13} \mathrm{Da}-$ her erscheint es aus sozialpolitischen Gründen grundsätzlich richtig, dass die Versicherteninformation auch weiterhin - wie von den Krankenkassen angegeben - über die Nutzung „klassischer Medien“ wie Kundenzeitschriften oder persönliche Ansprachen erfolgt. $\mathrm{Da}$ die bislang einzige existierende, aber schon mehr als 10 Jahre zurückliegende Studie zum Kenntnisstand der Versicherten hinsichtlich der Härtefallregelungen damals deutliche Defizite ermittelt hatte (vgl. Eller et al. (2002), S. 569 ff.), wäre es allerdings sinnvoll, den heutigen Kenntnisstand aus Versichertensicht nochmals zu erheben, um so eventuell noch vorhandene, aktuelle Defizite des Informationsverhaltens der Krankenkassen besser identifizieren und abstellen zu können.

Für die Mehrzahl der Krankenkassen stellt das aktuelle Antragsverfahren gegenüber einem „automatisierten“ Verfahren, das für jeden Versicherten unabhängig von einer Antragstellung die Anspruchsberechtigung überprüft, ein verwaltungsökonomisch effizienteres Verfahren dar. Problematisch daran ist aber, dass Versicherte eventuell aus Unkenntnis oder Scham keinen Antrag stellen und daher keinen Befreiungsstatus erhalten, obwohl sie eigentlich berechtigt wären (sog. „Dunkelziffer“). Ein „automatisiertes“ Verfahren könnte zwar helfen diese „Dunkelziffer“ zu vermeiden und wäre daher unter rein sozialpolitischen Aspekten zu bevorzugen. ${ }^{14}$ Allerdings spricht der sehr hohe administrative Aufwand dagegen, zumindest

\section{Ein Großteil der Krankenkassen präferiert eine Wiedereinführung der Sozialklausel.}

solange die administrativen Vorgänge nicht tatsächlich automatisiert sind, bspw. wie von einigen Krankenkassen vorgeschlagen durch eine Software, die Zuzahlungs- und Einkommensinformationen systemseitig aus den vorhandenen Daten generiert.

Die von einem Großteil der Krankenkassen präferierte Wiedereinführung des $\int 61$ SGB V i.d.F. bis Ende 2003 hätte zur Folge, dass viele der bislang nach der Chronikerklausel befreiten chronisch Kranken zukünftig wieder unter die Sozialklausel fallen würden. Hintergrund ist, dass zahlreiche chronisch Kranke auch Geringverdiener sind, ${ }^{15}$ die somit die Voraussetzungen für beide Härtefallklauseln erfüllen würden und dadurch die Möglichkeit hätten, sich für die ökonomisch vorteilhaftere Variante $\mathrm{zu}$ entscheiden. Umgekehrt führte daher die seinerzeitige Abschaffung der Sozialklausel im Rahmen des GMG zu einer sehr starken Zunahme der Härtefälle nach der Chronikerklausel. ${ }^{16}$ Die Sozialklausel verstößt nach Ansicht von Kritikern allerdings gegen die vertikale Gleichbehandlung (vgl. RürupKommission (2003), S. 12), da Geringverdiener vollständig von Zuzahlungen ausgenommen und damit prozentual weniger belastet wären als die übrigen Härtefallgruppen. Andererseits könnte es unter Verteilungsaspekten und in Anwendung des Solidarprinzips politisch gewollt sein, dass Geringverdiener nicht nur absolut, sondern auch prozentual gegenüber anderen Härtefallgruppen besser gestellt werden. Eine Wiedereinführung der Sozialklausel wäre zwar einerseits mit Mehrausgaben für die Krankenkassen verbunden. Diese müssten die von den Versicherten nicht zu tragenden Selbstbehalte (sog. „entgangene Zuzahlungen“) übernehmen, wodurch die Finanzierungsfunktion der Zuzahlungen reduziert wäre. ${ }^{17}$ Andererseits würde die oben skizzierte deutliche Verschiebung in den Härtefallgruppen (mehr Sozialklauselfälle, weniger Chronikerklauselfälle) zu wesentlich niedrigeren administrativen Kosten führen, da die Prüfung nach der Sozialklausel lediglich eine Einkommensprüfung umfasst. Demgegenüber stellt die Überprüfung des Chronikerstatus die umfangreichste Prüfung der Härtefallklauseln dar.

Eine weitere „Optimierungsmöglichkeit" besteht für zahlreiche Krankenkassen in der Festlegung einer einheitlichen Belastungsgrenze für alle Härtefälle. Dabei erscheinen die Gleichbehandlung aller Versicherten sowie die Reduktion des Verwaltungsaufwandes aufgrund der entfallenden Überprüfung des Chronikerstatus als vorteilhaft. Zudem bedeutet eine einheitliche Belastungsgrenze von $1 \%$ eine pekuniäre Besserstellung derjenigen Versicherten, die bislang unter die Überforderungsklausel fielen und mit 2\% ihrer Bruttoeinnahmen zum Lebensunterhalt belastet waren. Die Mehrkosten für die Krankenkassen aufgrund höherer entgangener Zuzah-

\section{ine einheitliche Belastungsgrenze würde den dministrativen Aufwand (ntlich reduzieren.}

lungen fallen aber nur für eine sehr kleine Versichertengruppe an (ca. 450.000 Versicherte) und können deshalb nach Einschätzung vieler Krankenkassen durch die dann entfallenden, administrativ sehr aufwändigen Prüfungen nach der Chronikerklausel (für ca. 6,78 Mio. Versicherte) kompensiert werden. Eine

13 So waren in Deutschland laut (N)ONLINER Atlas - trotz deutlich zunehmender Tendenz in den letzten Jahren - auch 2012 nur etwa $28 \%$ der über 70 -jährigen Internetnutzer, in der Altersgruppe der 60- bis 69Jährigen lag dieser Anteil 2012 auch nur bei etwas über 60\% (vgl. Initiative D21 (2012)).

14 „Automatisierte“ Prüfverfahren der Anspruchsberechtigung sind - wenn auch in anderem Kontext - in anderen Ländern durchaus im Einsatz. So werden im Kopfpauschalensystem der Schweiz die sozialpolitisch notwendigen Prämienverbilligungen in 7 Kantonen von den zuständigen Behörden „automatisch“ ohne Antragstellung ermittelt (vgl. Dennig et al. (2010), S. 44 f.). Auch in Deutschland soll der notwendige Sozialausgleich bei Erhebung der einkommensunabhängigen Zusatzbeiträge „automatisch“ ohne Antragstellung ermittelt werden (vgl. GKV-SV, DRV, BA (2011), S. 1 ff.). Faktisch ist dieses Verfahren aber bislang noch nicht zur Anwendung gekommen, da die relevante Rechengröße „durchschnittlicher Zusatzbeitrag" sowohl 2011, 2012 als auch 2013 von der Bundesregierung mit 0 EUR festgesetzt wurde (vgl. GKV-SV (o. J.(b))).

15 vgl. dazu auch den bekannten und bspw. in Lampert, Mielck (2008) beschriebenen Zusammenhang zwischen sozialer und gesundheitlicher Lage.

16 So stieg die Anzahl der Härtefälle nach der Chronikerklausel von 2,17 Mio. im Jahr 2002 (Zahlen für 2003 sind nicht verfügbar) auf 5,97 Mio. im Jahr 2004 (vgl. GBE (o.J.(a)), BMGS (2003)).

$17 \mathrm{Ob}$ es durch die Wiedereinführung der Sozialklausel auch zu einer Reduktion der Steuerungsfunktion von Zuzahlungen käme, ist fraglich, da schon die Annahme einer steuernden Wirkung von Zuzahlungen in der Literatur umstritten ist (vgl. dagegen bspw. Holst (2008), S. 12 ff. bzw. dafür bspw. Breyer, Zweifel, Kifmann (2013), S. 294) 
einheitliche Belastungsgrenze von $2 \%$ würde zwar auf jeden Fall eine positive Finanzierungswirkung hervorrufen, da von den bislang nach der Chronikerklausel befreiten Versicherten zukünftig Zuzahlungen i.H.v. 2\% ihrer Bruttoeinnahmen zum Lebensunterhalt zu leisten wären. Für chronisch Kranke würde sich dadurch aber eine deutliche ökonomische Schlechterstellung ergeben. Zudem könnten speziell chronisch Kranke mit niedrigem Einkommen davon abgehalten werden, erforderliche Gesundheitsleistungen in Anspruch zu nehmen (vgl. Pfaff et al. (2002), S.74).

Die Untersuchung hat des Weiteren gezeigt, dass alle antwortenden Krankenkassen ihren Versicherten die Möglichkeit der Vorabbefreiung anbieten. Davon sehen 43,2\% die Vorabbefreiung für alle Versicherten vor. Diese Praxis ist konform mit den Vorstellungen der ehemaligen Spitzenverbände der Krankenkassen, die aus Gründen der Gleichbehandlung die Regelung nicht nur auf ausgewählte Personenkreise beschränken wollen (vgl. Deutscher Bundestag (2006), S. 1 f.). Im Gegensatz dazu bieten mehr als die Hälfte der Krankenkassen $(56,8 \%)$ nur spezifischen Versichertengruppen die Vorabbefreiung an, was eher mit den Vorstellungen des Bundesgesundheitsministeriums (BMG) übereinstimmt, das keinen Widerspruch in der Begrenzung der Gewährung von Vorabbefreiungen auf nur bestimmte Personenkreise (bspw. Heimbewohner) und dem Gleichheitsgrundsatz sieht. Als Begründung führt das BMG an, dass nur auf diesem Weg steuernde Effekte von Zuzahlungen zu gewährleisten seien. Trotz der angeführten grundsätzlichen Bedenken akzeptiert das BMG aber die Praxis der Vorabzuzahlungsbefreiungen aller Versicherten (vgl. Deutscher Bundestag (2006), S. 1 f.).

Als weitere Optimierungsmöglichkeit wurde zudem von einigen Krankenkassen die Abschaffung der, ihrer Ansicht nach, „unsinnigen Vorsorgeklausel“ gefordert. Ursprünglich beinhaltete das Gesetz i.d.F. bis zum 09.04.2013 die Inanspruchnahme von Früherkennungsuntersuchungen nach $\mathbb{S} 25$ Abs. 1 SGB V ("Check-up 35“) und \25 Abs. 2 SGB V (Früherkennungsuntersuchungen zu Brust-, Darm- oder
Gebärmutterhalskrebs) als Voraussetzung zur Gewährung einer reduzierten Belastungsgrenze. Als Alternative für die Inanspruchnahme von Krebsfrüherkennungsuntersuchungen wurde vom G-BA in der Chroniker-Richtlinie eine einmalige Beratung über Chancen und Risiken der jeweiligen Untersuchungen als ausreichend definiert. Hintergrund war, dass bei der Inanspruchnahme von Krebsfrüherkennungsuntersuchungen mögliche Fehlbefunde (falsch-positiv/ falsch-negativ) mit entsprechend negativen Folgewirkungen nicht auszuschließen waren. Auf Basis einer analogen Argumentation wurde im erst kürzlich in Kraft getretenen Krebsfrüherkennungs- und -registergesetz (KFRG) die entsprechende Gesetzesvorschrift ersatzlos gestrichen (vgl. Deutscher Bundestag (2012), S. 33), wodurch auch die Festlegungen des G-BA zur einmaligen Beratung obsolet geworden sind. Nach dem Willen des Gesetzgebers soll das Inanspruchnahmeverhalten der einzelnen Versicherten zukünftig nicht mehr durch Anreizsysteme beeinflusst werden.

Weiterhin ihre Gültigkeit behalten haben aber die Regelungen zum „Checkup 35“ in $\$ 25$ Abs. 1 SGB V. In diesem Zusammenhang ist die Vorgabe einer Altersgrenze für Gesundheitsuntersu-

Folglich hätten Krankenkassen mit überdurchschnittlich vielen Härtefällen weiterhin einen Wettbewerbsnachteil.

chungen zu hinterfragen. Versicherte, die der gesetzlich festgelegten Altersgrenze nicht unterliegen und keine derartige Untersuchung durchführen lassen, fallen bei Auftreten einer chronischen Erkrankung nicht unter die höhere Belastungsgrenze von 2\% und bleiben somit von „Sanktionen“ unberührt. In diesem Zusammenhang wäre eine Aufhebung der Altersgrenze als Lösungsansatz denkbar, womit dem Gleichheitsgrundsatz Rechnung getragen wäre. Der Gleichheitsgrundsatz wird zudem in einem weiteren zu kritisierenden Punkt beschränkt (vgl. Gerlach (2009), S. 22 ff.). So ist es fragwürdig, ob die Teilnahme an strukturierten Behandlungsprogrammen (DMP) „die Sanktion wegen nicht in Anspruch ge- nommener Gesundheitsuntersuchungen eliminiert“ (Gerlach (2009), S. 24), denn strukturierte Behandlungsprogramme bestehen nicht für alle chronischen Erkrankungen.

Wie bereits dargestellt, sieht die Mehrzahl der Krankenkassen den Kassenwettbewerb durch die Härtefallregelungen aufgrund der einheitlichen gesetzlichen Regelungen bzw. Verwaltungsvorgaben nicht beeinflusst. Aus leistungsrechtlicher Sicht ist dieses Argument nachvollziehbar, dennoch stellt sich die Frage der Wettbewerbsrelevanz unter den Bedingungen des morbiditätsorientierten Risikostrukturausgleichs (Morbi-RSA). Zunächst ist festzuhalten, dass die von den Versicherten aufgrund der Härtefallregelungen nicht zu leistenden Zuzahlungen von den Krankenkassen übernommen werden (sog. „entgangene Zuzahlungen“). Da die Anteile an Härtefällen zwischen den einzelnen Krankenkassen variieren, sind sie auch in unterschiedlichem Ausmaß durch die sog. „entgangenen Zuzahlungen“ finanziell belastet. Hinzu kommt, dass auch die deutlichen Morbiditätsunterschiede zwischen Härte- und Nicht-Härtefällen (vgl. Langer (2005), S. 247) zu ungleichmäßigen Belastungen der Krankenkassen führen, zumal der Härtefallstatus bislang nicht explizit im RSA berücksichtigt wird, obwohl er einen nicht unbedeutenden Erklärungsfaktor für Ausgabenunterschiede darstellt (vgl. Langer (2005), S. 245). Der 2009 eingeführte M-RSA federt sicherlich Teile dieser Belastungen besser ab als der RSA vor 2009, da es eine Korrelation zwischen den im M-RSA berücksichtigungsfähigen Krankheiten (mit entsprechenden Zuschlägen) und dem Härtefallstatus, insbesondere von chronisch Kranken, geben dürfte. Allerdings besteht hinsichtlich des genauen Ausmaßes noch entsprechender Forschungsbedarf, sodass, wie schon in der Vergangenheit, von unterschiedlichen finanziellen Belastungen der Krankenkassen aufgrund der Härtefallregelungen - und somit einer gewissen Wettbewerbsrelevanz - auszugehen ist. Folglich hätten Krankenkassen mit überdurchschnittlich vielen Härtefällen weiterhin einen Wettbewerbsnachteil. Zudem würden für alle Krankenkassen nach wie vor bestimmte Anreize zur Risikoselektion von Härtefällen bestehen. 


\section{Literatur}

BMG (o. J.): Bundesministerium für Gesundheit (Hrsg.): Statistik KM6 zum 01.07.2012. Url.:http:// www.bmg.bund.de/fileadmin/dateien/ Downloads/Statistiken/GKV/Mitglieder Versicherte/KM6_2012_121120.xls [12.02.2013].

BMGS (2003): Bundesministerium für Gesundheit und Soziale Sicherung (Hrsg.): Gesetzliche Krankenversicherung. Leistungsfälle und -tage 2002 (Ergebnisse der GKV-Statistiken $\mathrm{KG}_{2} / 2002$ und $\mathrm{KG} 5 / 2002$ ), Berlin.

Breyer, F.; Zweifel, P.; Kifmann, M. (2013): Gesundheitsökonomik, Berlin u.a..

Coca, V.; Schröder, H. (2012): Ökonomische Aspekte des deutschen Arzneimittelmarktes 2011, in: Schwabe, U.; Paffrath, D. (Hrsg.): Arzneiverordnungs-Report 2012, Berlin u.a., S. 167-221.

Dennig, M.-C.; Greß, S.; Jacobs, K.; Reinhart, M. (2010): Der Sozialausgleich zur Flankierung von Zusatzbeiträgen im Licht von Erfahrungen aus der Schweiz, in: Gesundheits- und Sozialpolitik, Nr. 6, S. 41-48

Deutscher Bundestag (2006): Bericht der Spitzenverbände der Krankenkassen über die Praxis der Vorabzuzahlungsbefreiungen durch die Krankenkassen, Bundestagsdrucksache 16/2549, Berlin.

Deutscher Bundestag (2012): Entwurf eines Gesetzes zur Weiterentwicklung der Krebsfrüherkennung und zur Qualitätssicherung durch klinische Krebsregister (Krebsfrüherkennungs- und -registergesetz-KFRG), Bundestagsdrucksache 17/11267, Berlin.

Eller, M.; Baumann, H.; Mielck, A. (2002): Bekanntheitsgrad der Härtefallregelungen in der gesetzlichen Krankenversicherung, in: Das Gesundheitswesen, Nr. 11, S. 565-571.

Eller, M.; Holle, R.; Reitmeir, P.; John, J.; Nagl, H.; Mielck, A. (2004): Die Härtefallregelungen innerhalb der Gesetzlichen Krankenversicherung - wie viele Versicherte sind anspruchsberechtigt? Ergebnisse aus der Einkommensund Verbrauchsstichprobe 1998, in: Sozialer Fortschritt, Nr. 1, S. 1-7

Frese, E. (2005): Grundlagen der Organisation: entscheidungsorientiertes Konzept der Organisationsgestaltung, Wiesbaden.

G-BA (2007): Gemeinsamer Bundesausschuss (Hrsg.): Bericht der Arbeitsgruppe Zuzahlung des UA Prävention zur Konkretisierung des Regelungsauftrages aus $\$ 62$ Abs. I Satz 8 SGB V (Feststellung „therapiegerechten Verhaltens“), Berlin.

G-BA (2008): Gemeinsamer Bundesausschuss (Hrsg.): Richtlinie des Gemeinsamen Bundesausschusses zur Umsetzung der Regelungen in $\S 62$ für schwerwiegend chronisch Erkrankte („Chroniker-Richtlinie“), Berlin.
GBE (o. J.(a)): Gesundheitsberichterstattung des Bundes (Hrsg.): Überschreitungen der Belastungsgrenze für Zuzahlungen gemäß $\S 62$ SGB V der Versicherten der gesetzlichen Krankenversicherung. Url.: http:// www.gbe-bund.de/oowa921-install/servlet/oowa/aw92/dboowasys921.xwdevkit/ xwd_init?gbe.isgbetol/xs_start_neu/\&p aid=3\&p_aid $=35997256 \&$ nummer $=281 \& p_{-}$ sprache $=$ D\&p_indsp $=-\& p \_$aid $=72303220$ [11.01.2013]

GBE (o. J.(b)): Gesundheitsberichterstattung des Bundes (Hrsg.): Zuzahlungen der privaten Haushalte in der gesetzlichen Krankenversicherung. Url.: http://www. gbe-bund.de/oowa921-install/servlet/ oowa/aw92/dboowasys921.xwdevkit/ xwd_init?gbe.isgbetol/xs_start_neu/\&p aid=3\&p_aid $=35997256 \&$ nummer $=664 \& p$ sprache $=D \& p$ indsp $=-\& p$ aid $=66508326$ [11.01.2013]

Gerlach, W. (2009): $§ 62$ Belastungsgrenze, in: Hauck, K.; Noftz, W. (Hrsg.): Sozialgesetzbuch SGB V - Gesetzliche Krankenversicherung Kommentar. Erg.-Lfg. II/og, K § 62, Berlin.

GKV-SV (o. J.(a)): GKV-Spitzenverband (Hrsg.): Alle gesetzlichen Krankenkassen. Url.: http://www.gkv-spitzenverband.de/ krankenversicherung/krankenversicherung_ grundprinzipien/alle_gesetzlichen krankenkassen/alle gesetzlichen krankenkassen.jsp [15.10.2012].

GKV-SV (o. J.(b)): GKV-Spitzenverband (Hrsg.): Zusatzbeitrag. Url.: http://www. gkv-spitzenverband.de/krankenversicherung/ krankenversicherung_grundprinzipien/ finanzierung/zusatzbeitrag/zusatzbeitrag.jsp [31.03.2013].

GKV-SV (2010): GKV-Spitzenverband (Hrsg.) Verfahrensgrundsätze zur Vorschrift über die Erstattung bzw. Befreiung von gesetzlichen Zuzahlungen gemäß $\S 62$ Abs. 1, 2 und 3 SGB V vom 4./ 5.10.2010. Url.: http://www vdek.com/content/vdeksite/vertragspartner/ leistungen/zuzahlung_befreiung/ verfahrensgrundsaetze_62_sgbv.pdf [15.03.2013].

GKV-SV; DRV; BA (2011): GKV-Spitzenverband; Deutsche Rentenversicherung Bund; Bundesagentur für Arbeit (Hrsg.): Beitrags- und melderechtliche Auswirkungen des Sozialausgleichs nach $\S 242 b$ SGB V. Url.: http://arbeitgeber.barmer-gek.de/ barmer/web/Portale/Arbeitgeberportal/ Arbeitshilfen 2ound 20Formulare/Vorschrifte n_20_26_20Praxishilfen/PDF/Rundschreiben/ Sozialausgleich.pdf [29.01.2013].
Holst, J. (2008): Kostenbeteiligung für Patienten: Reformansatz ohne Evidenz; theoretische Betrachtungen und empirische Befunde aus Industrieländern. Überarbeitete und aktualisierte Fassung des WZB Discussion Papers SP I 2007-304, Wissenschaftszentrum Berlin für Sozialforschung, Berlin.

Initiative D21 (2012): (N)ONLINER Atlas 2012 Basisdaten für Deutschland. Url.: http:// www.initiatived21.de/portfolio/nonliner-atlas [24.02.2013]

Kieser, A.; Walgenbach, P. (2010): Organisation, Stuttgart.

Krummaker, S.; Mittendorf, T.; Stürmann, H.; Graf von der Schulenburg, J.-M. (2008): Entwicklungen des gesetzlichen Krankenversicherungsmarktes in Deutschland: Ergebnisse einer empirischen Erhebung, in: Gesundheits und Sozialpolitik, Nr. 6, S. 28-39.

Lampert, T.; Mielck, A. (2008): Gesundheit und soziale Ungleichheit - Eine Herausforderung für Forschung und Politik, in: Gesundheit und Gesellschaft Wissenschaft, Nr. 2, S. 7-16.

Langer, B. (2005): Steuerungsmöglichkeiten des GKV-Arzneimittelmarktes - Selbstbeteiligungen unter besonderer Berücksichtigung von Härtefallregelungen, Berlin.

Langer, B.; Pfaff, A.B.; Kern A.O. (2006): Steuerung des GKV-Arzneimittelmarktes Auswirkungen von Selbstbeteiligungen und Härtefallregelungen, in: Volkswirtschaftliche Diskussionsreihe der Universität Augsburg, Nr. 285, Augsburg.

Langer, B.; Pfaff, A.B.; Rindsfüßer, C.; Eller, M.; Mielck, A.; Baumann, H. (2003): Berücksichtigung von Härtefällen im Rahmen des Risikostrukturausgleichs?, in: Gesundheits- und Sozialpolitik, Nr. 5-6, S. 43-50.

Pfaff, M.; Pfaff, A.B.; Langer, B.; Mielck, A.; Eller, M. (2002): Härtefallregelungen nach SGB V $\$ \$ 61 \mathrm{f}$.: Art, Umfang und Folgen von tatsächlicher Inanspruchnahme und potentieller Berechtigung, Gutachten im Auftrag des Bundesministeriums für Bildung und Forschung in Kooperation mit der AOK Bayern, der Verrechnungsstelle Süddeutscher Apotheken (VSA) und der GSF - Forschungszentrum für Umwelt und Gesundheit, Augsburg.

Rürup-Kommission (2003): Geschäftsstelle der Kommission für die Nachhaltigkeit in der Finanzierung der Sozialen Sicherungssysteme (Hrsg.): Selbstbeteiligung in der Gesetzlichen Krankenversicherung, Berlin.

SVRG (2012): Sachverständigenrat zur Entwick lung im Gesundheitswesen (Hrsg.): Wettbewerb an der Schnittstelle zwischen ambulanter und stationärer Gesundheitsversorgung. Sondergutachten 2012, Bundestagsdrucksache 17/10323, Berlin. 